\title{
Implementación de un sistema de riego con recuperación de agua proveniente de la climatizacion en el sector Industrial
}

\section{Implementation of an irrigation system with water recovery from climate control in the Industrial sector}

FUENTES-RUBIO, Yadira Aracely†**, ESQUEDA-WALLE, Ramiro, DOMÍNGUEZ-CRUZ, René Fernando y SALAZAR-CASTILLO, Yaaresi Milliset

Universidad Autónoma de Tamaulipas

ID $1^{\text {er }}$ Autor: Yadira Aracely, Fuentes-Rubio / ORC ID: 0000-0002-7385-9794, CVU CONACYT ID: 794463

ID $1^{\text {er }}$ Coautor: Ramiro, Esqueda-Walle / ORC ID: 0000-0003-2838-5216, CVU CONACYT ID: 270645

ID $2^{\text {do }}$ Coautor: René Fernando, Domínguez-Cruz / ORC ID: 0001-7001-7543, Researcher ID Thomson: C-3108-2018, CVU CONACYT ID: 31057

ID $3^{\text {er }}$ Coautor: Yaaresi Milliset, Salazar-Castillo / ORC ID: 0000-0001-6424-0298

DOI: $10.35429 /$ JOIE.2019.10.3.24.30

Recibido 16 de Abril, 2019; Aceptado 30 de Junio, 2019

\section{Resumen}

En este trabajo se presenta el estudio y análisis del uso del agua generada por las unidades de aire acondicionado en una empresa manufacturera de productos electrónicos para la industria médica, industrial $\mathrm{y}$ automotriz. La empresa conoce su responsabilidad ambiental y está consciente de que el agua es necesaria para la vida, por ello, se debe utilizar adecuadamente y cuidarla, para evitar que este recurso no renovable se siga agotando. Se propuso utilizar el agua que se genera por la condensación de los aires acondicionados ubicados en tres áreas de la empresa. El proyecto, permitió recuperar y reusar los 2,104,400 litros de agua generados anualmente en dichos equipos de refrigeración mediante el diseño e implementación de un sistema funcional de riego con cobertura de $820 \mathrm{~m}^{2}$ de césped. El uso del agua para este sistema de riego se encuentra basado entre otros elementos, en el mantenimiento de los aires acondicionados, lo que evita la generación de excedentes de químicos contaminantes en agua condensada que se desaloja.

Mantenimiento Industrial, Reutilización de agua, Responsabilidad Ambiental

\begin{abstract}
This paper presents the study and analysis of the use of water generated by air conditioning units in a company that manufactures electronic products for the medical, industrial and automotive industry. The company knows its environmental responsibility and is aware that water is necessary for life, therefore, it must be used and taken care of, to avoid that this resource is not renewable. We propose to use the water generated by the condensation of air conditioners in the company's areas. The project, which will recover and reuse the $2,104,400$ liters of water generated in said refrigeration equipment through the design and implementation of functional irrigation systems with a coverage of $820 \mathrm{~m} 2$ of grass. The use of water for this irrigation system is based on other elements, in the maintenance of the air conditioners, which avoids the generation of surplus chemical contaminants in the condensed water that is discharged.
\end{abstract}

Industrial Maintenance, Water Reuse, Environmental Responsibility

Citación: FUENTES-RUBIO, Yadira Aracely, ESQUEDA-WALLE, Ramiro, DOMÍNGUEZ-CRUZ, René Fernando y SALAZAR-CASTILLO, Yaaresi Milliset. Implementación de un sistema de riego con recuperación de agua proveniente de la climatizacion en el sector Industrial. Revista de Ingeniería Innovativa. 2019. 3-10: 24-30

*Correspondencia al Autor (Correo electrónico: yfuentes@ @at.edu.mx)
$\dagger$ Investigador contribuyendo como primer Autor. 


\section{Introducción}

La Organización de las Naciones Unidas para la Educación, la Ciencia y la Cultura ha señalado que factores como el crecimiento demográfico, la urbanización, la industrialización, el incremento de la producción de cultivos y el consumo, ocasionan un incremento en la demanda de agua potable (UNESCO, 2015). Así mismo, la concentración de gases de efecto invernadero en la atmósfera aumenta la temperatura y con ello se modifican diversas funciones del ecosistema, tales como el ciclo hidrológico, la disponibilidad hídrica, las propiedades de la composición y naturaleza del suelo y la vegetación, entre muchas otras (Intergovernmental Panel on Climate Change, $I P C C, 2014)$. Ello propicia que los veranos sean más intensos y la disponibilidad hídrica disminuye en zonas semiáridas, siendo necesario identificar las opciones para mitigar y afrontar estos efectos, así como explorar las estrategias ante los nuevos escenarios (Gray y Brady, 2016).

Con base en lo anterior, es necesario una gestión sostenible del agua y del cuidado del ecosistema, es por ello que las empresas de manufactura a nivel mundial se rigen y certifican en la norma ISO 14001. Esta norma tiene el propósito de apoyar la aplicación de un plan de manejo ambiental, la cual fue creada por la Organización Internacional para Normalización (International Organization for Standardization - ISO) y cuyo objetivo es que la empresa reduzca su impacto en el medio ambiente y con ello crear beneficios internos al mejorar el uso de los recursos.

Algunas empresas de manufactura, para obtener la certificación ISO 14001 se centran en desarrollar un plan de protección ambiental y cumplir con las leyes nacionales referentes al medio ambiente, mientras que, para otras, implica mucho más, tal es el caso de la empresa manufacturera ubicada en $\mathrm{Cd}$. Reynosa, Tamaulipas, la cual se dedica a la fabricación de productos electrónicos para la industria médica, industrial y automotriz.

Al Sistema de Gestión Ambiental en dicha empresa, se propuso desarrollar un proyecto para el cuidado del agua y utilización eficiente de la misma, el cual permitiría el regado de las áreas verdes, debido a que estas zonas se incrementaron.
El objetivo se enfocó en la recuperación y reutilización de 2,104,400 litros de agua generados anualmente en equipos de refrigeración, mediante el diseño e implementación de sistema funcional de riego con una cobertura de $820 \mathrm{~m}^{2}$ de césped. Ello fue posible mediante el desarrollo de un plan de mantenimiento a los aires acondicionados para evitar la generación de excedentes de químicos contaminantes en agua condensada que se desaloja.

Más allá del tema y caso de análisis, la relevancia de la investigación se sustenta principalmente por las siguientes consideraciones; i) el contexto y tendencias de la región en la que se ubica la empresa; ii) las características sectoriales de su actividad productiva. Respecto a las primeras se tiene que Reynosa es el municipio más poblado del estado con 630,000 habitantes y en los últimos 25 años se ubica dentro de los primeros cinco municipios a nivel nacional con las mayores tasas de crecimiento promedio anual poblacional y dentro de los primeros diez de la producción bruta total (Censos Poblacionales y Económicos de INEGI, varios años). En este sentido el dinamismo de la actividad manufacturera de exportación -principalmente- ha contribuido en esta tendencia y de manera paralela al acelerado aumento de la demanda de suministro de agua potable que conforme a estimaciones propias con datos de SIMBAD-INEGI y CEAT (varios años), ha registrado una expansión de $16 \%$ de 2006 a 2018 y las tomas industriales se han quintuplicado de 1996 a la fecha.

\section{Nociones fundamentales del estudio}

\section{Norma ISO 14001:2015}

La Organización Internacional de Normalización (ISO), menciona que el propósito de ISO 14001 en su versión 2015, es proporcionarles a las organizaciones un marco de referencia para proteger el medio ambiente y responder a las condiciones ambientales cambiantes, en equilibrio con las necesidades socioeconómicas.

Un enfoque sistemático a la gestión ambiental proporciona información a la alta dirección para generar éxito a largo plazo y crear opciones para contribuir al desarrollo sostenible, en el presente artículo, nos centraremos en: 
1. La protección del medio ambiente, por medio de la prevención o mitigación de impactos ambientales adversos;

2. Mejora del desempeño ambiental;

3. El control o la influencia sobre la forma en la que la organización diseña, fabrica, distribuye, consume y lleva a cabo la disposición final de productos $\mathrm{o}$ servicios, usando una perspectiva de ciclo de vida que pueda prevenir que los impactos ambientales sean involuntariamente trasladados a otro punto del ciclo de vida;

4. El logro de beneficios financieros y operacionales que puedan ser el resultado de implementar alternativas ambientales respetuosas que fortalezcan la posición de la organización en el mercado;

La base para el enfoque de un sistema de gestión ambiental se fundamenta en el concepto de Planificar, Hacer, Verificar y Actuar (PDCA, por sus siglas en inglés), es decir, el circulo de Deming. Adicionalmente, Gutiérrez (2010), menciona que la filosofía de este ciclo lo hace de gran utilidad para perseguir la mejora mediante diferentes metodologías.

La Organización Internacional de la Normalización (ISO), menciona que en el marco de referencia introducido en la Norma Internacional ISO 14001, se puede integrar en el modelo PCDA, lo cual puede ayudar a usuarios actuales y nuevos a comprender la importancia de un enfoque de sistema.

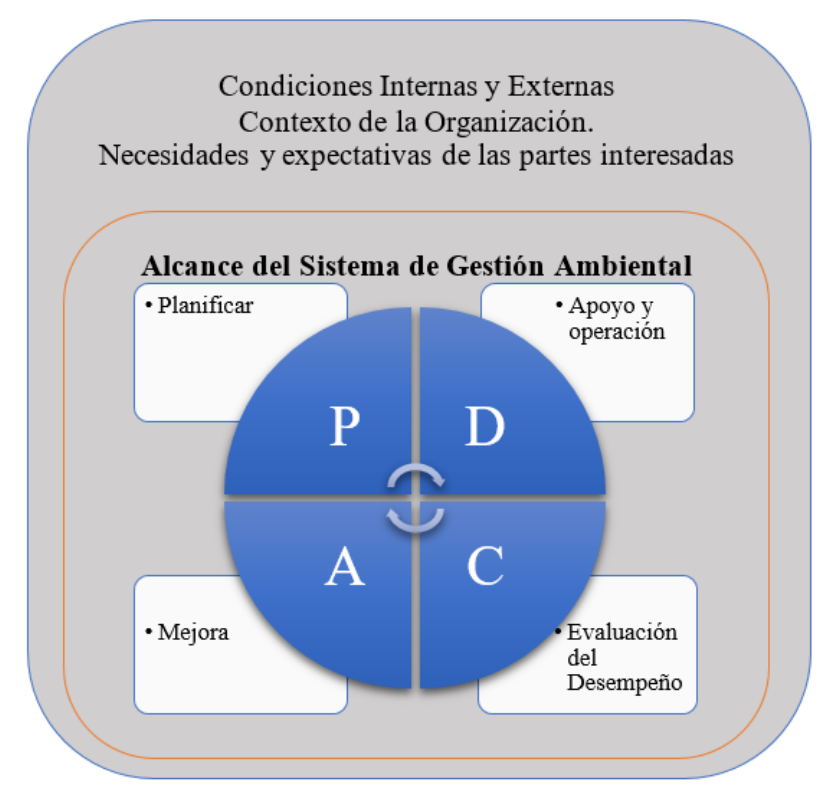

Figura 1. Circulo de Deming y su relación con el SGA. Fuente: Realización Propia
Sistema de gestión ambiental:

La Organización Internacional de Normalización (ISO), lo conceptualiza como: parte del sistema de gestión usada para gestionar aspectos ambientales, cumplir los requisitos legales y otros requisitos, así como abordar los riesgos y oportunidades.

\section{Mantenimiento industrial:}

Como lo menciona Peng (2012), dentro de las organizaciones, cuando escuchamos el término "mantenimiento" lo asociamos al departamento encargado de la reparación y preservación de los equipos de producción, sin embargo, dentro de la literatura de la administración del mantenimiento, el término es por lo general definido como el conjunto de acciones técnicas y administrativas encaminadas a mantener o restaurar un bien capital a una condición optima esperada.

Existen tres tipos de mantenimiento básicos, mencionados por Dhillon (2002), los cuales son el mantenimiento predictivo, el preventivo y el correctivo, sin embargo, el artículo se centra en el mantenimiento preventivo. El mantenimiento preventivo tiene por misión mantener un nivel de servicio determinado en los equipos, programando las intervenciones de sus puntos vulnerables en el momento más oportuno. Suele tener un carácter sistemático, es decir, se interviene, aunque el equipo no haya dado ningún síntoma de tener un problema.

\section{Problemática}

La empresa manufacturera en cuestión decidió instalar nuevas áreas verdes dentro y fuera de las instalaciones de la empresa. Al realizar el riego a dichas áreas se notó que el consumo de metros cúbicos y el costo del agua incrementó notablemente y esto afectó la meta de los métricos ambientales con un incremento del $30 \%$ en su totalidad. Aproximadamente se consumían 2600 litros de agua mensuales en el sistema de riego, lo cual representaba un consumo anual de 30,830 litros. 


\section{Metodología}

En la empresa se tienen instalados aires acondicionados industriales de 50 toneladas, los cuales anualmente generan 2,104,400 litros de agua condensada, y debido al incremento en el consumo de agua para el riego de las nuevas aéreas verdes, se reunieron los departamentos de Mantenimiento de planta con el de Seguridad y medio ambiente, entre ambos trabajaron en el proyecto para reutilizar el agua condensada, utilizando la metodología del círculo de Deming como lo establece la ISO 14001:2015 para el sistema de gestión ambiental.Se acordaron las siguientes actividades para desarrollo del proyecto:

1. Desarrollar un plan de mantenimiento preventivo a los aires acondicionados que evite la contaminación en el agua condensada.

2. Analizar el agua arrojada por los aires acondicionados y dicha agua debe estar bajo la norma Mexicana NOM-127SSA1-1994 - Salud ambiental. Agua para uso y consumo humano, límites permisibles de calidad y tratamientos a que debe someterse el agua para su potabilización.

3. Implementación del sistema de riego utilizando el agua condensada.

1. Desarrollo del plan de mantenimiento:

Un aire acondicionado se compone de dos unidades: interior y exterior, tal como se muestran en la figura 2 . a)

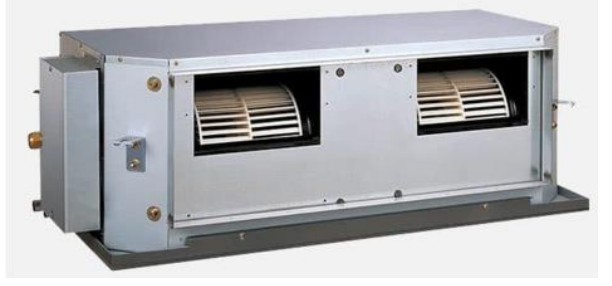

b)

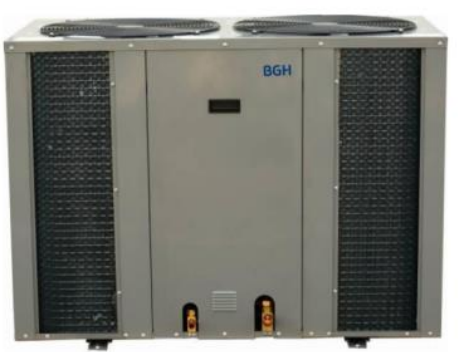

Figura 2. Unidades que conforman un aire acondicionado industrial. a) Unidad Interna. b) Unidad Externa Fuente: https://www.bgh.com.ar/producto/l39/separadocomercial-frio-calor-por-bomba

En la unidad exterior se encuentra el intercambiador (ubicado en el compresor), cuando este se enciende se llena de gas a baja presión y la temperatura dentro de él desciende, a través de este proceso, la humedad ambiental se va adhiriendo al intercambiador y así se produce la condensación. Entonces, el gas se convierte en agua y esta cae a la bandeja del desagüe (figura 3).

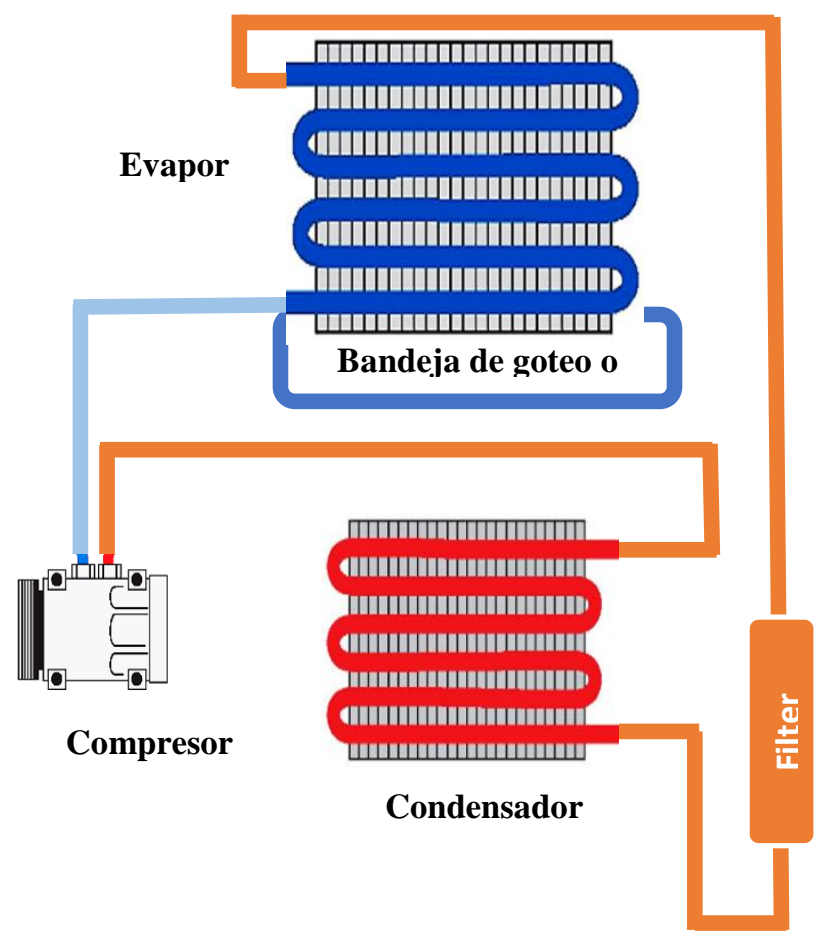

Figura 3. Ciclo básico de refrigeración

Fuente: Realización Propia 
Dependiendo de la humedad que haya en el ambiente se condensa de mayor o menor cantidad. La época del año en la que se aprovecha mejor dicho proceso de condensación es en verano esto se debe a que en esta época del año hay más humedad. El mantenimiento es una pieza clave para el buen funcionamiento de la captación de agua para su posterior reutilización, de no ejecutarlo correctamente podría ocasionar un impacto ambiental negativo, es por ello que se desarrollaron los planes de mantenimiento preventivo al sistema de climatización que a continuación se presentan:

\section{Mantenimiento Preventivo:}

\begin{tabular}{|l|l|}
\hline \multicolumn{1}{|c|}{ Actividades } & Frecuencia \\
\hline $\begin{array}{l}\text { Limpieza de equipo y partes, el } \\
\text { condensador y evaporador. }\end{array}$ & Semanal \\
\hline $\begin{array}{l}\text { Lubricación a los mecanismos (baleros, } \\
\text { chumaceras y bujes). }\end{array}$ & Mensual \\
\hline $\begin{array}{l}\text { Revisión y cambio de filtros en unidades } \\
\text { paquetes. }\end{array}$ & Mensual \\
\hline Cambio de Filtro deshidratador. & Mensual \\
\hline $\begin{array}{l}\text { Revisión de amperaje y voltaje de } \\
\text { compresor bombas y ventiladores, }\end{array}$ & 6 meses \\
\hline $\begin{array}{l}\text { Analizar las muestras de agua condensada } \\
\text { según lo indique la NOM-127. }\end{array}$ & 6 meses \\
\hline Limpieza del tanque de almacenamiento. & Anual \\
\hline
\end{tabular}

Tabla 1. Tareas del mantenimiento preventivo para aire acondicionado

Fuente: Realización Propia

\section{Análisis del agua condensada:}

Después de la implementación del plan de mantenimiento, se realizó el análisis del agua condensada, y se compararon los resultados con los límites permitidos en base a la norma NOM127-SSA1-1994 (Tabla 2). El análisis del agua lo realizó un laboratorio especializado, para una mayor confiabilidad de los resultados y posterior reutilización del agua.

\begin{tabular}{|l|r|r|}
\hline \multicolumn{1}{|c}{ Características } & \multicolumn{1}{c|}{$\begin{array}{c}\text { Agua } \\
\text { condensada } \\
\text { analizada }\end{array}$} & $\begin{array}{r}\text { Límites Permisibles } \\
\text { (NOM-127-SSA1- } \\
\text { 1994) }\end{array}$ \\
\hline $\mathrm{Ph}$ & 6.34 & $6.5-8.5$ \\
\hline $\begin{array}{l}\text { Solidos } \\
\text { Suspendidos }\end{array}$ & $30 \mathrm{mg} / \mathrm{l}$ & 1000.00 \\
\hline Bario & $0.02 \mathrm{ppm}$ & 0.70 \\
\hline Magnesio & $0 \mathrm{ppm}$ & 0.15 \\
\hline Sulfato & $0 \mathrm{ppm}$ & 400.00 \\
\hline Densidad & $0.9983 \mathrm{~g} / \mathrm{cm} 3$ & 500.00 \\
\hline Cloruros & $106.5 \mathrm{ppm}$ & $0.2-1.50$ \\
\hline Dureza de Calcio & $30 \mathrm{ppm}$ & $500.00 \mathrm{ppm}$ \\
\hline Dureza Total & $40 \mathrm{ppm}$ & $500.00 \mathrm{ppm}$ \\
\hline Dureza de Magnesio & $10 \mathrm{ppm}$ & $0.15 \mathrm{ppm}$ \\
\hline
\end{tabular}

Tabla 2. Resultados del análisis del agua condensada. Fuente: Realización Propia

\section{Implementación del sistema de riego:}

Se instaló el sistema de riego para cubrir un total de $820 \mathrm{~m}^{2}$, en donde básicamente el agua condensada es llevada a un tinaco y de ahí mediante una bomba, el agua es llevada mediante mangueras y rociadores (figura 4) a todas las áreas verdes, como se muestra en la figura 5 .

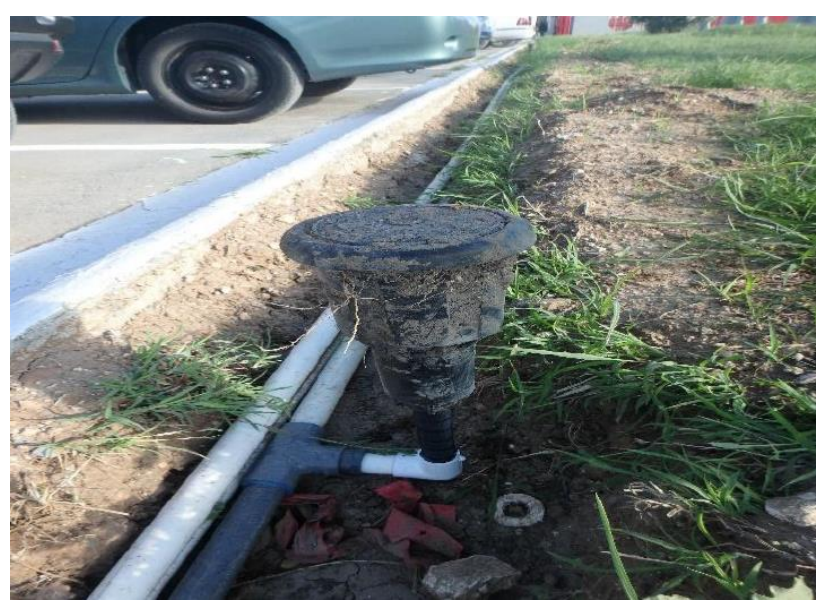

Figura 4. Rociadores del sistema de riego Fuente: Realización Propia

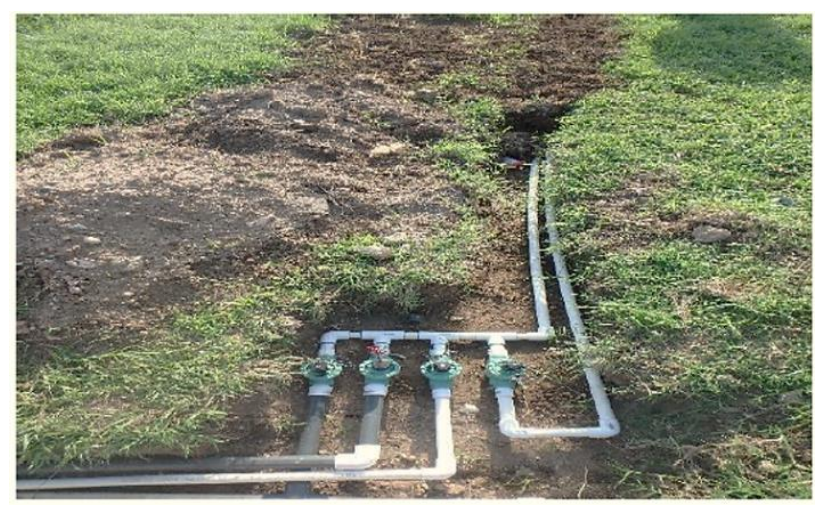

Figura 5 Instalación del sistema de riego

Fuente: Realización Propia

\section{Resultados}

Se continúo utilizando el agua condensada de los aires acondicionados, siguiendo la metodología propuesta y después de 12 meses, se registró el consumo mensual utilizado en el sistema de riego, esto equivale a un ahorro en promedio de poco más de 2500 litros mensuales, tal como se muestra en la gráfica 1. 


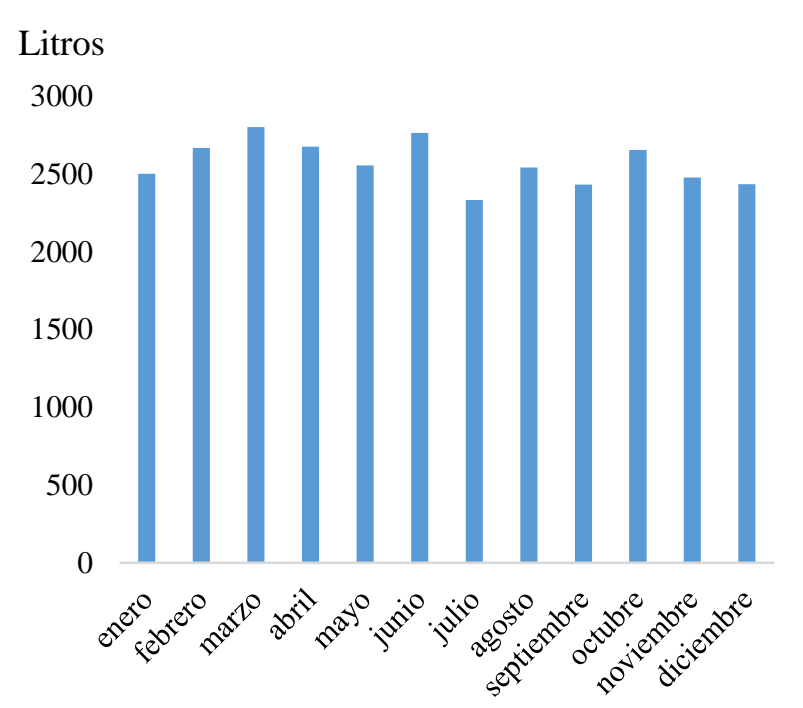

Gráfico 1. Consumo mensual de agua utilizada en el sistema de riego

Fuente: Realización Propia

Según el Periódico Oficial del Estado de Tamaulipas (POE), en el municipio de Reynosa la tarifa de agua por cuota mínima (10 metros cúbicos) es de $\$ 55.57$ para uso residencial y público, \$106.42 comercial y \$133.58 industrial, en base a ello, al reutilizar el agua condensada de los aires acondicionados, se está obteniendo registra un ahorro monetario significativo, como se muestra en la gráfica 2.

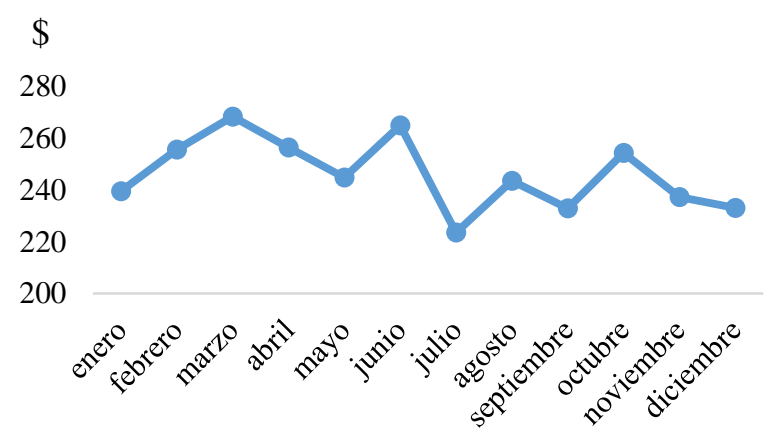

Gráfico 2. Ahorro mensual

Fuente: Realización Propia

En promedio el ahorro mensual fue de $\$ 247.57$, lo que en términos anuales asciende a $\$ 3,000$. El beneficio en términos de la Gestión de la Calidad en el apartado ambiental y, sobre todo, en la contribución a la sustentabilidad de la región son muy superiores a los económicos y con un beneficio potencialmente mucho mayor, ya que es importante mencionar que no todos los litros de agua recuperados están siendo utilizados en el sistema de riego. Por tanto, la empresa está analizando implementar un segundo proyecto para utilizar esa agua en los sanitarios y de esta manera seguir contribuyendo con el medio ambiente.

\section{Conclusiones}

De los resultados obtenidos, se puede concluir que el agua que proviene de los aires acondicionados es un recurso que por su cantidad y calidad llevando a cabo el mantenimiento en tiempo y forma, cumple con los lineamientos de la Norma Mexicana para el uso de aguas y con ello puede utilizarse sin problemas para el riego, lo cual es muy favorable y ambientalmente sostenible. Adicionalmente, se observó que la recuperación y uso del agua generada por los sistemas de aire acondicionado permitió un ahorro del $51 \%$, lo que genera además de un beneficio económico, una contribución al cuidado en los recursos hídricos. Este proyecto ha propiciado que el uso del agua generada de los aires acondicionados pueda también ser empleada en otras áreas, como en los sanitarios, tal como la empresa proyecta incorporarla a futuro.

\section{Recomendaciones}

En términos regionales el impacto de este tipo de implementaciones cobra una dimensión mayor tomando en cuenta que conforme a los datos más recientes de Index -Reynosa existen 130 plantas manufactureras de exportación instaladas en Reynosa por lo que, bajo un escenario conservador si se pusiera en marcha proyectos similares en cada una de ellas se estarían reutilizando aproximadamente 3,120,000 litros anuales de agua potable lo que podría ayudar a contener las tendencias de la demanda de agua de uso industrial señaladas al inicio del trabajo.

\section{Referencias}

Bain, R., R. Cronk, "Global Assessment of Exposure to Faecal Contamination through Drinking Water Based on a Systematic Review", doi.org/10.1111/tmi.12334, Tropical Medicine and Inter. Health, 19 (8), 917-27 (2014)

Comisión Estatal de Agua del Estado de Tamaulipas, CEAT. https://www.tamaulipas.gob.mx/ceat/ [Fecha de consulta: Mayo, 2019]

Dhillon, B.S. Engineering Maintenance: A Modern Approach; CRC Press: Boca Raton, Florida, Estados Unidos, 2002. 
Gray, S. y S. Brady, "Plant developmental responses to climate change, Developmental Biology”, 419(1), 64-77 (2016)

Gutierrez, Humberto. Calidad Total y Productividad. Mexico. McGraw-Hill. Mexico (2010).

International Organization for Standardization (2019). "ISO 14001:2015”, https://www.iso.org/obp/ui\#iso:std:iso:14001:e d-3:v1:es/ [Fecha de consulta: Junio, 2019]

INEGI. Censos Generales de Población y Vivienda (varios años); Censos Económicos (varios años). www.inegi.org.mx [Fecha de consulta: Abril, 2019]

Intergovernmental Panel on Climate Change. Climate Change 2014. Syntesis Report

IPCC. Climate Change 2014: Synthesis Report. Contribution of Working Groups I, II and III to the Fifth Assessment Report of the Intergovernmental Panel on Climate Change [Core Writing Team, R.K. Pachauri and L.A. Meyer (eds.)]. 151 pp. PCC, Génova, Suiza (2014).

International Organization for Standardization (2019). "ISO 14001:2015", https://www.iso.org/obp/ui\#iso:std:iso:14001:e d-3:v1:es/ [Fecha de consulta: Junio, 2019] Index-Reynosa. http://indexreynosa.org.mx/ Fecha de consulta: Mayo, 2019]

Organización de las Naciones Unidas para la Educación, la Ciencia y la Cultura, "Informe Mundial sobre el Desarrollo de los Recursos Hídricos de las Naciones Unidas 2015: Agua para un mundo sostenible", UNESCO, París, 139 p. (2015)

Organización Mundial de la Salud, (OMS), "Agua para todos, agua para la vida, ler Informe de las Naciones Unidas sobre el desarrollo de los recursos hídricos en el mundo", París (2015)

Peng, Kern, (2012). “Equipment Management in the post-maintenance era: a new alternative to Total Productive Maintenance (TPM)". CRC Press. Estados Unidos de América.

Periódico Oficial del Estado de Tamaulipas. http://po.tamaulipas.gob.mx/wp-
content/uploads/2019/04/cxliv-49-230419F.pdf [Fecha de consulta: AgostMayo, 2019]

Norma Oficial Mexicana NOM-127-SSA11994, "Salud ambiental. Agua para uso y consumo humano. Límites permisibles de calidad y tratamientos a que debe someterse el agua para su potabilización". (2000). http://www.salud.gob.mx/unidades/cdi/nom/m1 27ssa14.html [Fecha de consulta: Junio, 2019]

SIMBAD-INEGI. Estadísticas sobre urbanización (varios años). [Fecha de consulta: Abril, 2019] 